Check for updates

Cite this: RSC Adv., 2018, 8, 14237

Received 15th March 2018

Accepted 25th March 2018

DOI: 10.1039/c8ra02258e

rsc.li/rsc-advances

\section{Recent progress in biochar-supported photocatalysts: synthesis, role of biochar, and applications}

\begin{abstract}
Md Manik Mian (D) ab and Guijian Liu*ab
Incorporating photocatalytic nanoparticles with biochar templates can produce biochar-supported photocatalysts (BSPs) and combine the advantages of biochar with catalytic nanoparticles. The obtained composite exhibits excellent surface properties, crystallinity, chemical stability, recoverability, and higher photocatalytic competency than the bare semiconductor photocatalyst. The literature and advances in BSPs based on the combination of low-cost biochar and catalytic nanoparticles are presented in this review. Various synthetic techniques and physicochemical properties of BSPs are summarized. The article then discusses in detail the important role of biochar in influencing the photocatalytic performance of BSPs such as supporting nanoparticles, increasing the surface area and the number of active sites, shuttling electrons, acting as an electron reservoir, increasing charge separation, and reducing band gap energy. Furthermore, the synergistic effects of adsorption and photodegradation of organic pollutants by BSPs are discussed with in-depth mechanistic evidence. Finally, the application of BSPs in various fields and constructive suggestions for their future development are reported.
\end{abstract}

\section{Introduction}

Nowadays, environmental pollution and insufficient sources of natural clean energy are serious global problems faced by humankind. The high growth rate of the world's population, industrialization, and urbanization accelerates the consumption of non-renewable energy and causes the release of pollution into the air and waterways. According to a UN water report, on average $70 \%, 28-38 \%$, and $8 \%$ municipal wastewater is treated before discharge in high-income, middle-income, and low-income countries, respectively, and about $80 \%$ of total global wastewater is discharged into the environment without any treatment. ${ }^{1}$ Untreated or improperly treated wastewater discharges certainly impose a detrimental effect on the natural ecosystem and public health. Therefore, a diverse group of researchers devoted themselves to find an environmentally friendly, cost-effective, and sustainable way to resolve this adverse situation. From that perspective, photocatalysis, in which naturally available, safe, and clean solar energy has been utilized by semiconductor photocatalysts to degrade harmful organic pollutants, is a major advancement.

Up until now, various nanoparticles such as $\mathrm{RuO}_{2}, \mathrm{TiO}_{2}$, $\mathrm{Fe}_{2} \mathrm{O}_{3}, \mathrm{SiO}_{2}, \mathrm{Al}_{2} \mathrm{O}_{3}, \mathrm{ZrO}_{2}, \mathrm{ZnO}$, CdS, and $\mathrm{ZnS}$ have been utilized

${ }^{a}$ CAS Key Laboratory of Crust-Mantle Materials and Environments, School of Earth and Space Sciences, University of Science and Technology of China, Hefei 230026, China. E-mail: lgj@ustc.edu.cn; Fax: +86 551 63621485; Tel: +86 55163603714

${ }^{b}$ State Key Laboratory of Loess and Quaternary Geology, Institute of Earth Environment, The Chinese Academy of Sciences, Xi'an, Shaanxi 710075, PR China as semiconductor photocatalysts. ${ }^{2-6}$ Among them, different forms of $\mathrm{TiO}_{2}$ nanoparticles have received significant attention due to their chemical stability, low toxicity, inexpensiveness, availability, and high sensitivity to UV light illumination. ${ }^{7}$ However, the large band gap energy and the separation of $\mathrm{TiO}_{2}$ nanoparticles are the major drawbacks for their applications. Furthermore, $\mathrm{TiO}_{2}$ cannot utilize most of the solar energy spectrum due to its large band gap energy. ${ }^{4}$ Therefore, an external UV light source is required for their activation, which is not a cost-effective method. However, various hybrid composites synthesized by combining doping of transition metals (Ti, $\mathrm{Fe}, \mathrm{Ru}, \mathrm{Au}$, and $\mathrm{Mo})^{8-11}$ and/or non-metals $(\mathrm{C}, \mathrm{N} \text {, and } \mathrm{S})^{\mathbf{1 2 - 1 4}}$ can improve the photocatalytic performance, but, high preparation costs, non-renewability, and risks of secondary pollution prevent their large-scale application. Therefore, more and more attention has been applied to giving a stable platform for photocatalytic nanoparticles by incorporating secondary materials, which can improve the recoverability and visible light sensitivity of the photocatalysts. Biochar, as a supporting material for catalytic nanoparticles, receives significant attention in this direction. The unique physicochemical properties of biochar are beneficial for incorporating various photocatalytic nanoparticles. ${ }^{2,15}$

Biochar is a low-cost, stable, environmentally friendly, and sustainable material produced from available waste biomass via pyrolysis, hydrolysis, gasification, and carbonization methods. ${ }^{16,17}$ The prominent fractions of plant- and animaloriginated biomass used for biochar production are illustrated in Fig. 1. The major streams of biomass are lignocellulose, starch, 


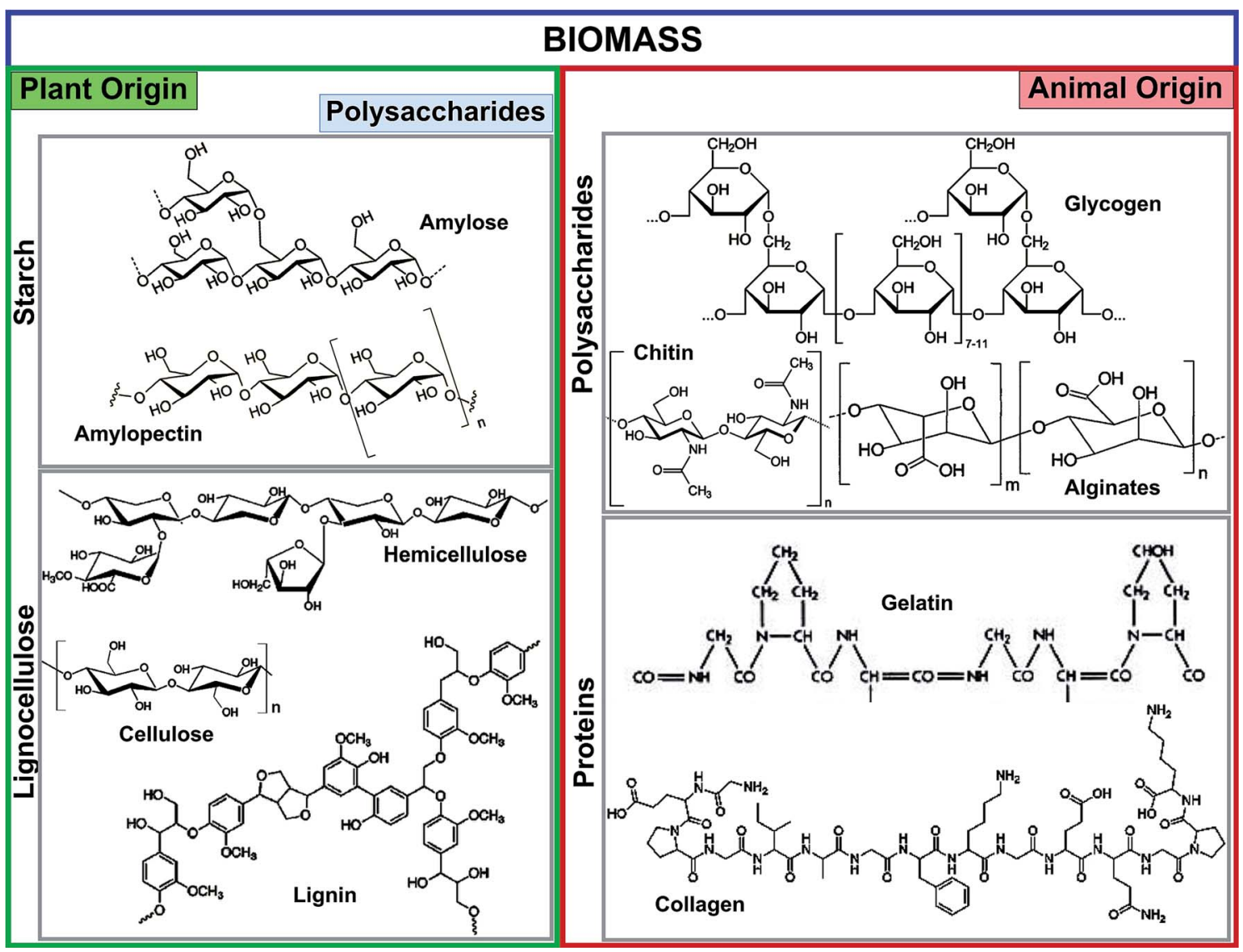

Fig. 1 Prominent fractions of biomass feedstocks derived from plants and animals.

animal protein, and polysaccharides. Starch and lignocellulose are the forms of plant-driven biomass used for the synthesis of biochar and different chemicals. Starch is composed of a large number of glucose units connected by glycosidic bonds and consists of two molecules such as amylose and amylopectin. Starch is commonly used as a pore-forming agent in ceramic technology. ${ }^{18}$ The composition of lignocellulose, especially lignin and hemicellulose structures, is highly dependent on its origins such as hardwood, softwood, or grasses. Lignin is a constituent of the cell wall, and is the second most abundant polymer after cellulose. Lignin, composed of three different phenyl propane monomers, is electronegative and has a strong affinity for various electropositive metal ions. ${ }^{2}$ Among animal-originated polysaccharides, chitin is the most abundant natural polysaccharide, and can be found in the exoskeleton of insects and crustacean cell walls. ${ }^{19}$ The amino (chitin, chitosan, gelatin, and collagen) and hydroxyl (chitin, chitosan, and glycogen) functional groups of animal-origin biochar possess strong adsorption capacities for various heavy metals ${ }^{20}$ dyestuffs, ${ }^{20}$ and pathogens. ${ }^{21}$ Moreover, the $-\mathrm{OH}$ and $-\mathrm{NH}_{2}$ functional groups of biomass can easily be tuned with other functional materials.

Biochar can be used as an excellent platform for supporting various catalytic nanoparticles due to its unique surface properties, easily tunable functional groups, chemical stability, and electrical conductivity. ${ }^{22-24}$ The electron-conductive nature of biochar can reduce the quick recombination of the $\mathrm{e}^{-} / \mathrm{h}^{+}$pair during photocatalysis ${ }^{25}$ and suitable surface functional groups enable immobilization of different pollutants, which is favorable for photocatalysis. ${ }^{26,27}$ An excellent review article published recently describes the $\mathrm{TiO}_{2}$-immobilized carbon from different renewable sources and introduces the application of biochar in the synthesis of photocatalysts as well. ${ }^{2}$ Consequently, various biochar-supported photocatalysts have been synthesized and reported in recent years. The reported BSPs show promising improvements in visible light sensitivity, chemical stability, recollection ability, and photocatalytic degradation of various pollutants. Some of those recently reported BSPs are $\mathrm{TiO}_{2}-\mathrm{SPW}$, $\mathrm{TiO}_{2}$-MSP, ${ }^{28} \mathrm{TiO}_{2}$-waste plum, ${ }^{29} \mathrm{TiO}_{2}$-corn cob, ${ }^{25,30} \mathrm{TiO}_{2}$-chitosan, ${ }^{31} \mathrm{TiO}_{2}$-wheat husks, ${ }^{32} \mathrm{TiO}_{2}$-bamboo, ${ }^{33} \mathrm{TiO}_{2}$-reed straw, ${ }^{34}$ g- $\mathrm{C}_{3} \mathrm{~N}_{4} / \mathrm{FeVO}_{4}$-pine needles, ${ }^{35} \mathrm{ZrO}_{2}$-wheat husks, ${ }^{36} \mathrm{~g}_{-}-\mathrm{C}_{3} \mathrm{~N}_{4}{ }^{-}$ chestnut, ${ }^{37} \mathrm{TiO}_{2}$-wood charcoal, ${ }^{38}$ and BiOX-biochar. ${ }^{39}$

We believe that it is high time to give a comprehensive review on the recent advancements of biochar-supported photocatalysts, in particular, on their syntheses, applications, functionality, and future prospects based on environmental sustainability. Therefore this review provides major scientific insight into BSPs synthesized from low-cost biochar combined with catalytic nanoparticles, summarizes their syntheses and physicochemical properties, discusses in depth the role of biochar in BSPs driving photocatalysis, highlights their applications in various fields, and provides useful suggestions for the future development of efficient and novel BSPs. 


\section{BSP synthesis methods}

\subsection{Sol-gel}

Sol-gel is the most common method for synthesizing BSPs. In $\mathrm{TiO}_{2}$-BSP, granular-shaped anatase and/or rutile $\mathrm{TiO}_{2}$ is agglomerated on the biochar surface via a sol-gel synthetic method. ${ }^{25}$ The crystal phase structure, average size, and dispersion of $\mathrm{TiO}_{2}$ upon the biochar depends on the sol-gel decomposition temperature. Usually, calcination temperatures below $700{ }^{\circ} \mathrm{C}$ can create anatase $\mathrm{TiO}_{2}$ crystals. The dispersion of $\mathrm{TiO}_{2}$ and changes in biochar structure increase with the calcination temperature. ${ }^{34}$ At high temperatures above $700{ }^{\circ} \mathrm{C}, \mathrm{TiO}_{2}$ starts forming the rutile crystalline structure and the percentages of rutile increase with the rising temperature. ${ }^{40}$ BSP synthesis through sol-gel methods shows significant alteration of oxygen-containing functional groups due to their involvement in $\mathrm{TiO}_{2}$ doping with biochar. A typical sol-gel method follows three sequential formation steps: (1) prepare biochar templates via thermal decomposition of biomass, (2) increase surface oxides and reduce $\mathrm{pH}$ of biochar via acid treatment, then deposit catalytic nanoparticles on the biochar surface, and finally (3) calcine the obtained biochar-laden catalytic nanoparticles to give a stable structure..$^{25,34,41}$ The schematic of the BSP synthetic process using the sol-gel method is illustrated in Fig. 2A. Recently, Zhang et al. ${ }^{34}$ synthesized an efficient $\mathrm{TiO}_{2}-$ reed straw BSP using the sol-gel method for the photodegradation of sulfamethoxazole. They produced the biochar template by heating at a temperature of $500{ }^{\circ} \mathrm{C}$ for $6 \mathrm{~h}$ with a heating rate of $20{ }^{\circ} \mathrm{C} \mathrm{min}^{-1}$. The obtained biochar was crushed and sieved through a $0.15 \mathrm{~mm}$ mesh, and rinsed with $\mathrm{HCl}$ acid $\left(1 \mathrm{~mol} \mathrm{~L}^{-1}\right)$ for $2.5 \mathrm{~h}$ to increase the number of surface oxides and reduce the $\mathrm{pH}$ at the point of zero charge. The acidtreated biochar was used as the precursor for $\mathrm{TiO}_{2}$ doping.

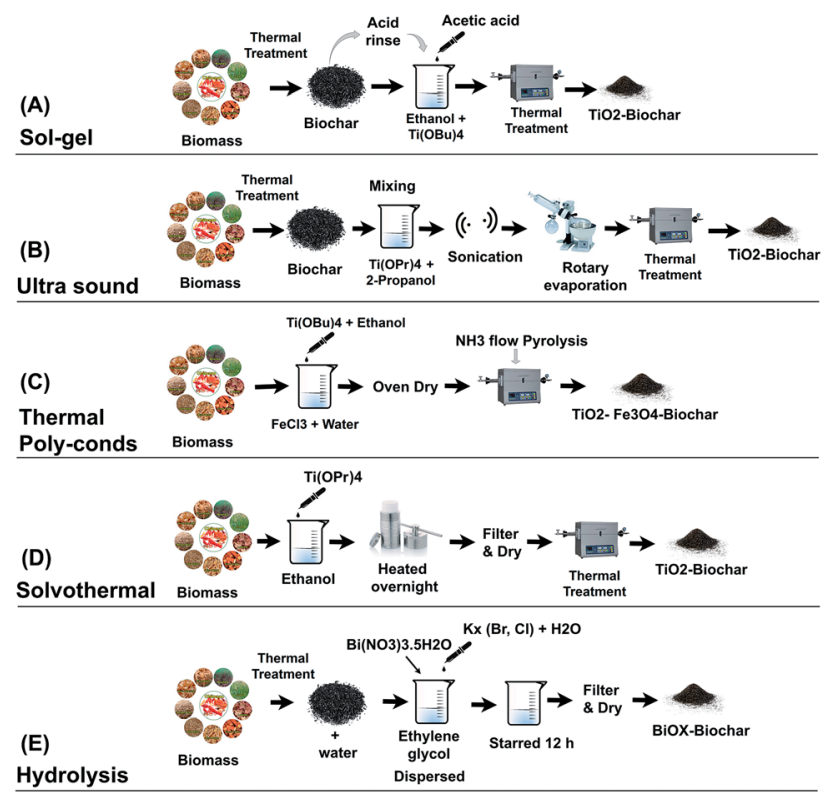

Fig. 2 Schematic of BSP synthetic methods followed by interpretation of ref. $28,31,34$ and 39 .
Later, the precursor was immersed in $50 \mathrm{~mL}$ ethanol and $20 \mathrm{~mL}$ $\mathrm{Ti}(\mathrm{OBu})_{4}$ solution, and $2.5 \mathrm{~mL}$ acetic acid was added dropwise into that solution. The prepared mixture was stirred for $2 \mathrm{~h}$ at room temperature. Then, $2.5 \mathrm{~mL}$ of ultra-pure water $(\mathrm{pH}=2)$ was added and stirring was continued for a further $4 \mathrm{~h}$. After stirring the obtained mixture, it was dried in an oven at $105{ }^{\circ} \mathrm{C}$. The dried sample of $\mathrm{Ti}(\mathrm{OBu})_{4}$-laden biomass was further calcined in a muffle furnace at different temperatures $\left(300-500{ }^{\circ} \mathrm{C}\right)$ to obtain the $\mathrm{TiO}_{2}$-biochar.

\subsection{Ultrasound}

Ultrasound (US) is a very new technique for the synthesis of BSPs. Recently, Lisowski et al. ${ }^{28}$ followed a multistep synthetic process with ultrasound mechanisms for the preparation of $\mathrm{TiO}_{2}-$ softwood pellets and $\mathrm{TiO}_{2}-$ miscanthus straw pellet biochar. The BSPs obtained contain enormous cracked structures on the surface that confirm the formation of a mesoporous structure. Using US treatment, anatase $\mathrm{TiO}_{2}$ crystal structures formed on the BSPs, and the growth of the $\mathrm{TiO}_{2}$ crystal size and structure is significantly higher than that of BSPs formed by a non-US method. A local trapping state due to $\mathrm{Ti}^{3+}$ below the $\mathrm{TiO}_{2}$ conduction band reduces the band gap of $\mathrm{TiO}_{2}$, and therefore the obtained material shows visible light sensitivity. US methods can act as interfacial mediators to improve the visible light response of the photocatalyst. The schematic of the multistep process with US mechanisms for the synthesis of BSPs is illustrated in Fig. 2B. Firstly, biochar was prepared through a thermal decomposition process at $500-700{ }^{\circ} \mathrm{C}$. Then, the obtained biochar was processed for further treatment by crushing, sieving, washing, and drying. The clean and dry biochar (500 g) was mixed with a titanium(Iv) isopropoxide and propanol solution, and that mixture was ultrasonicated for $1 \mathrm{~h}$. After sonication, the materials were extracted using a rotary vacuum evaporator assisted by sonication. The sonicated material was dried and further calcined at $400{ }^{\circ} \mathrm{C}$ to prepare the $\mathrm{TiO}_{2}$-biochar.

\subsection{Thermal polycondensation}

Thermal polycondensation is a simple and single-step heating synthesis of BSPs. Recently Liu et al. ${ }^{37}$ and our research group synthesized BSPs using a thermal polycondensation process. Liu et al. followed a multistep calcination process where biochar was obtained firstly from a thermal decomposition process. Then, the obtained biochar was blended with melamine in different ratios and ground. Later, the mixture of melaminebiochar was placed in a furnace and calcined at $300{ }^{\circ} \mathrm{C}$ for $2 \mathrm{~h}$ with a heating rate of $5{ }^{\circ} \mathrm{C} \mathrm{min}^{-1}$ for the synthesis of BSPs. However, our research group produced an agar biochar-based catalyst $\left(\mathrm{N}-\mathrm{TiO}_{2}-\mathrm{Fe}_{3} \mathrm{O}_{4}\right.$-biochar $)$ via a single-step thermal polycondensation process that is shown in Fig. 2C. The natural polysaccharide agar was used due to its thermally responsive sol-gel properties. At low temperatures $\left(30-40{ }^{\circ} \mathrm{C}\right)$, agar formed a gel network, which is beneficial for doping with other elements. ${ }^{\mathbf{4 2 , 4 3}}$ The nitrogen functional groups were activated on the catalyst via $\mathrm{NH}_{3}$ ambiance calcination. The obtained BSPS $\left(\mathrm{N}-\mathrm{TiO}_{2}-\mathrm{Fe}_{3} \mathrm{O}_{4}\right.$-biochar) had a significantly reduced band gap energy, and therefore, the material is able to perform excellent 
visible light photocatalysis for methylene blue degradation. It is well-known that the appropriate proportion of transition metal Fe- and N-doping with $\mathrm{TiO}_{2}$ nanoparticles can significantly reduce the band gap energy ${ }^{\mathbf{4}}$ and improve visible light photocatalysis. ${ }^{7,45,46}$ Furthermore, the $\mathrm{Fe}_{3} \mathrm{O}_{4}$ of the composite can activate the magnetic properties of the BSPs, which is suitable for easy collection from the aqueous media.

\subsection{Solvothermal}

$\mathrm{TiO}_{2}$-aggregated hollow spheres supported by carbon structures of furfural, saccharose, or chitosan can be prepared via a solvothermal method. Mainly anatase and some rutile $\mathrm{TiO}_{2}$ crystal structures formed on the composites through solvothermal synthesis. The crystal size of the sphere increased after calcination. The typical solvothermal synthesis follows several sequential steps (Fig. 2D). Firstly, catalytic nanoparticles such as $\mathrm{TiO}_{2}$ are deposited on the biomass templates via mixing them in an ethanol solution. For example, $1 \mathrm{~g}$ of carbon supporting materials such as furfural, ${ }^{47}$ chitosan, or saccharose $^{31,48,49}$ and $1 \mathrm{~g}$ of titanium(Iv) isopropoxide are dissolved in $18 \mathrm{~mL}$ of ethanol. Secondly, the prepared solution is sealed into a Teflon-lined stainless steel autoclave followed by solvothermal treatment at $\sim 175{ }^{\circ} \mathrm{C}$ overnight. The resultant solid after treatment is collected by filtration, washed several times, and dried. Finally, that dry sample is calcined at $600-800{ }^{\circ} \mathrm{C}$ for $6 \mathrm{~h}$ to remove the inner materials and form $\mathrm{TiO}_{2}$ hollow spheres. Composites synthesised by solvothermal methods show a reduction in the band gap energy and an improvement in visible light photocatalysis.

\subsection{Hydrolysis}

Some researchers have reported the synthesis of BSPs using a simple hydrolysis process, and improvement in visible light sensitivity during photocatalysis of organic pollutants. Min et $a .^{39}$ incorporated $\mathrm{BiOX}(\mathrm{X}=\mathrm{Br}$ or $\mathrm{Cl})$ with biochar through a hydrolysis method for the degradation of methyl orange. The schematic for the hydrolysis process for the synthesis of BSPs is given in Fig. 2E. Firstly, $2 \mathrm{mmol} \mathrm{Bi}\left(\mathrm{NO}_{3}\right)_{3} \cdot 5 \mathrm{H}_{2} \mathrm{O}$ was dispersed in $20 \mathrm{~mL}$ ethylene glycol at ambient temperature. Meanwhile, preprepared biochar was evenly dispersed into $20 \mathrm{~mL}$ pure water by sonication. Later, the biochar solution was added dropwise into the $\mathrm{Bi}\left(\mathrm{NO}_{3}\right)_{3} \cdot 5 \mathrm{H}_{2} \mathrm{O}$ solution. Afterwards, a pre-prepared $\mathrm{KX}$ (Br, Cl) solution ( $2 \mathrm{mmol} \mathrm{KX}$ with $20 \mathrm{~mL}$ pure water) was poured slowly into the mixture. The prepared mixture was stirred for a further $12 \mathrm{~h}$ at ambient temperature. Finally, the solid residue of $\mathrm{BiOX}(\mathrm{Br}, \mathrm{Cl})$-biochar in the mixture was collected, washed, dried, and stored for further use. Except for the heating required for biochar production, further heating is not required for BSP synthesis by the hydrolysis method, which is energy-saving.

\section{Role of biochar in BSPs and photocatalysis}

\subsection{Supporting nanoparticles}

Biochar can give an excellent platform for supporting a large number of nanoparticles. Simple thermochemically converted biochar contains available surface functional groups $(\mathrm{C}=\mathrm{O}, \mathrm{C}-$ $\mathrm{O}, \mathrm{COOH}, \mathrm{COO}-, \mathrm{OH}$ etc.) and an enormous pore structure. The surface functional groups of biochar can easily be reacted with other functional materials. Moreover, some metallic and nonmetallic mineral-containing biomass produced by thermal conversion shows the existence of $\mathrm{N}, \mathrm{S}, \mathrm{P}, \mathrm{K}, \mathrm{Ca}$, and $\mathrm{Mg}$ content in biochar, ${ }^{50}$ which can directly act as an adsorbent, catalyst, or catalyst support. ${ }^{51}$ A recent review article by Tan et al. ${ }^{52}$ stated the general overview of different nanoparticle incorporation with biochar templates, their synthesis, and applications for environmental pollution decontamination. The tuning of biochar with different nanoparticles improves biochar functionality and pollutant removal competency via adsorption and/or catalytic degradation. Nanoparticles can combine with biochar in two ways: (1) thermal decomposition of nanoparticle-loaded biomass, and (2) tuning nanoparticles with prepared biochar functional groups through chemical modification. For example, Yang et al. ${ }^{53}$ synthesized $\mathrm{Fe}_{3} \mathrm{O}_{4}$-deposited biochar via a single step pyrolysis of $\mathrm{FeCl}_{3}$-laden sawdust biochar. Initially, the $\mathrm{FeCl}_{3}$-laden biochar hydrolyzed to form $\mathrm{Fe}(\mathrm{OH})_{3}$ and $\mathrm{FeO}(\mathrm{OH})$ during the drying process. After subjecting the $\mathrm{FeCl}_{3}$ biomass to pyrolysis, the biomass components such as lignocellulose decomposed quickly into volatile components and biochar. Meanwhile, $\mathrm{FeO}(\mathrm{OH})$ was reduced to form $\mathrm{Fe}_{3} \mathrm{O}_{4}$ by the reducing agents $\mathrm{H}_{2}$, CO, and amorphous carbon which were produced by the pyrolysis reaction. The advantages of metal-laden biomass pyrolysis are: a single step heating process, simultaneously producing nanoparticle-loaded biochar, and metal compounds which can have some catalytic effect on biochar pyrolysis which improves bio-oil and biochar textural properties. ${ }^{22}$

In recent years, natural polysaccharides and their derivatives have received significant interest in the synthesis of biocharbased materials due to their availability, low toxicity, biocompatibility, biodegradability, and easy modifiability with various nanoparticles. ${ }^{54}$ In drug delivery systems, natural polysaccharides are commonly used for carrying nanoparticles. ${ }^{55}$ Among different natural polysaccharides, chitosan is the most widely used polysaccharide in different fields of research as well as for biochar production. ${ }^{56}$ The nanoparticles can incorporate with chitosan easily by beading, crosslinking, grafting, and surface impregnation. ${ }^{57}$ Chitosan is soluble in acidic water due to the protonation of $\mathrm{NH}_{2}$. Acid-soluble chitosan can turn back into a flock structure by adjusting the $\mathrm{pH}$ to 7.5-9 with $\mathrm{NaOH}$ due to deprotonation and insolubility of the polymer at natural $\mathrm{pH}^{56}$ Using this mechanism, an excellent iron-based $\gamma-\mathrm{Fe}_{2} \mathrm{O}_{3}$ biochar has been synthesized from water hyacinths and applied for the removal of $\mathrm{Cr}(\mathrm{vI}) .^{58}$ Recently, our research group used agar for the synthesis of a $\mathrm{N}-\mathrm{TiO}_{2}-\mathrm{Fe}_{3} \mathrm{O}_{4}$ doped photocatalyst. The gel matrix of agar at low temperature gives an excellent platform for supporting $\mathrm{TiO}_{2}$ and $\mathrm{Fe}_{3} \mathrm{O}_{4}$ nanoparticles. The enormous number of $\mathrm{OH}$ or $\mathrm{NH}$ functional groups in natural polysaccharides is easily tunable with the catalytic nanoparticles.

\subsection{Increasing surface area and number of active sites}

Usually, catalytic nanoparticles have a small surface area. When nanoparticles are dispersed on the biochar surface, the 
synthesized material (BSP) surface area can have an increased surface area compared to the bare nanoparticles. However, the overall surface area of biochar may decrease due to aggregation of metallic nanoparticles. ${ }^{37}$ Nanoparticles can homogeneously disperse on the biochar surface via suitable modification processes. Well-dispersed surface nanoparticles can improve light scattering and increase the number of active sites, which enhances photodegradation of organic pollutants. Lisowski et al. ${ }^{28}$ Balu et al. ${ }^{59}$ and Wang et al. ${ }^{60}$ stated that the larger surface area and adequate number of active sites of $\mathrm{TiO}_{2}$-biochar composites can lead to the materials exhibiting greater photocatalytic effects due to the presence of more photocatalytic surface-active site centers. Moreover, biochar templates can provide an aromatic carbon structure and different hydrophilic and hydrophobic surface functional groups that can favor adsorption of organic pollutants via $\pi-\pi$ stacking, hydrogen bonding, and/or electrostatic interactions. The higher adsorption competencies of the catalyst can facilitate the photodegradation. Nawi et al. ${ }^{61}$ reported that the synergies of adsorption-photodegradation of aqueous phenol by $\mathrm{TiO}_{2}$-chitosan can increase the degradation competency and reduce charge carrier recombination. Similar mechanisms were also reported by Zhang $e t ~ a l .{ }^{34}$ for photocatalytic degradation of sulfamethoxazole by $\mathrm{TiO}_{2}$-reed straw biochar. Biocharsupported $\mathrm{TiO}_{2}$ prevented $\mathrm{e}^{-} / \mathrm{h}^{+}$recombination and increased sulfamethoxazole photodegradation from $58.47 \%$ (commercial $\mathrm{TiO}_{2}$ ) to $91.27 \%$.

\subsection{Shuttling electrons}

Some amorphous biochars have semiconductor characteristics, which helps to improve the reactivity when they are incorporated with nanoparticles via transferring electrons. A large number of studies have provided significant evidence about biochar electrical conductivity in different systems. ${ }^{62}$ Biochars facilitated the electron transformation from a bulk chemical electron donor to the receptor. ${ }^{63,64}$ Biochar surface redox-active moieties mediate the mechanisms of electron shuttling between different reactants. The condensed aromatic ring structure of biochar can promote the electron transfer process. ${ }^{62,63} \mathrm{Wu}$ et al. ${ }^{65}$ stated that $\mathrm{Fe}_{3} \mathrm{O}_{4}$ nanoparticleincorporated carbonaceous gel networks derived from watermelon biomass allow continuous and stable shuttling of electrons and electrolyte ions to the electrode surface. The excellent electrochemical behavior of $\mathrm{Ag}$ nanoparticle-covered waste biochar was also reported by Yao and $\mathrm{Wu} .^{66}$ In the $\mathrm{TiO}_{2}-$ biochar or any BSP, the dense aromatic structure of biochar was able to shuttle electrons from the activation sites to the acceptors such as organic pollutants. This electron shuttling can reduce the quick recombination of $\mathrm{e}^{-} / \mathrm{h}^{+}$pairs and enhance the photodegradation of pollutants.

\subsection{Electron reservoir}

Biochar also can act as an electron reservoir. It is important to note that the electron storage and shuttling mechanisms of biochar are different processes. The electron shuttling process requires the conductive domain of black carbon (graphene) or the dense aromatic structure of biochar, while electron storage is the function of oxidation and reduction of biochar quinone moieties. A small number of studies have been conducted to investigate the electron reservoir potential of biochar. Klüpfel et $a{ }^{67}{ }^{67}$ reported that plant-based biochar has the capacity to store electrons up to $2 \mathrm{mmol} \mathrm{\textrm {e } ^ { - }}$ per $\mathrm{g}$. However, the electron storage capacity (ESC) of biochar depends on the types of biomass and the pyrolysis conditions. Biochar synthesized by intermediate to high heat temperatures shows a higher electron storage capacity via electron donation-acceptance. The electron donation and acceptance mechanisms by quinone moieties are illustrated in Fig. 3. Similar to humic acid, biochar has an enormous quinone content and can act as both electron donor and acceptor. Further, Saquing et al. ${ }^{68}$ found that wood-driven black carbon biochar has 0.85 and $0.87 \mathrm{mmol} \mathrm{e}^{-}$per $\mathrm{g}$ ESC in the acetate oxidation and nitrate reduction system. The electron sink capacity of biochar can be investigated by electron balance calculations. Saquing et al. showed that a portion of the electrons were missing during ethanol oxidation by Geobacter metallireducens, indicating the electron sink behaviour by the biochar. Xu et al. ${ }^{69}$ synthesized $\mathrm{TiO}_{2}$-carbon nanotubes (CNTs) and stated that CNTs can act as electron reservoirs during the photodegradation of benzene and methyl orange. In $\mathrm{TiO}_{2}$-biochar composites, carbon can act as an electron reservoir that conducts away $\mathrm{e}^{-}$from the $\mathrm{e}^{-} / \mathrm{h}^{+}$pair and activated $\mathrm{TiO}_{2}$ during photocatalysis. Acting as an electron reservoir increases the efficiency of charge separation from $\mathrm{TiO}_{2}$ and hinders the $\mathrm{e}^{-} / \mathrm{h}^{+}$ pair recombination. ${ }^{2}$

\subsection{Improving charge separation}

The charge separation due to the $\mathrm{e}^{-} / \mathrm{h}^{+}$pair is one of the important aspects of photocatalysis. Usually, bare semiconductor photocatalysts are activated by charge separation after light irradiation. However, they could recombine sooner with each other and achieve deactivation. In that case, a secondary component may maintain or even strengthen the activated state of the semiconductor photocatalyst, shown in reactions (R1)-(R4) following the interpretation of Shi. ${ }^{70}$

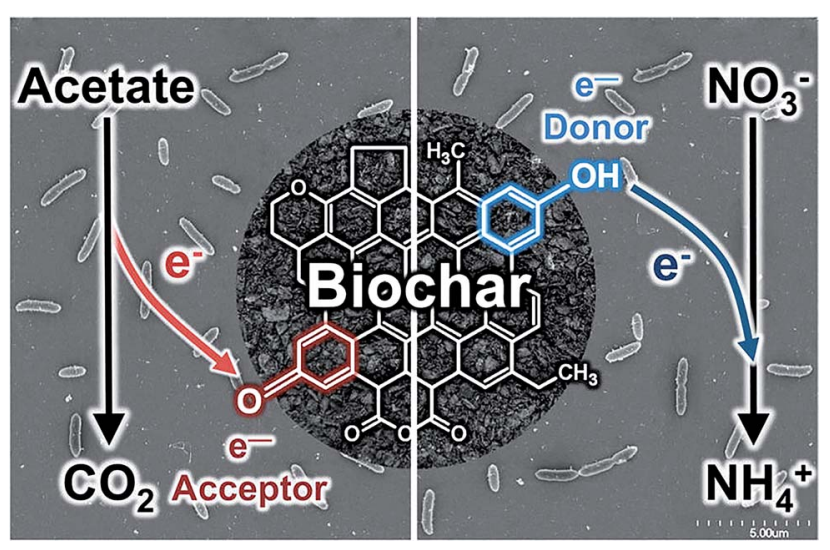

Fig. 3 Electron storage mechanisms of biochar via quinone moiety electron acceptance and donation. Reproduced with permission from ref. 68 . 


$$
\begin{gathered}
\mathrm{P} \rightarrow \mathrm{P}^{*} \\
\mathrm{P}^{*} \rightarrow \mathrm{P} \\
\mathrm{P}-\mathrm{C} \rightarrow \mathrm{P}^{*}-\mathrm{C}
\end{gathered}
$$

The single catalyst is activated after light irradiation (R1), while without any secondary element, the activated catalyst achieves deactivation again (R2). However, after a secondary element is incorporated into the catalyst, the activated state of the catalyst is well-maintained. Combination leads to activation of the catalyst (P) by other catalysts or non-catalysts (C) (R3). This accelerates the catalytic kinetic reaction and in some special cases both components can be mutually activated (R4).

$$
\mathrm{P}+\mathrm{C} \rightarrow \mathrm{P} * / \mathrm{C}^{*}
$$

In the BSP, electron shuttling from the catalytic nanoparticle to carbon significantly improves charge separation. The charge separation competency of the photocatalyst can be detected by photoluminescence (PL) spectroscopy and the photocurrent response. The lower PL spectrum intensity and the higher photocurrent density indicates the higher efficiency of the electron-hole pair separation. ${ }^{28,39}$ Lisowski et al. ${ }^{28}$ synthesized $\mathrm{TiO}_{2}$-biochar through a simple ultrasound sonication process and indicated that the charge separation significantly increases when biochar is incorporated with $\mathrm{TiO}_{2}$. The formation of heterojunctions between the $\mathrm{TiO}_{2}$ and biochar templates is responsible for the photoinduced charge separation. The similar increase in charge separation due to $\mathrm{Fe}$ and $\mathrm{FeVO}_{4}$ heterojunctions in the $\mathrm{g}-\mathrm{C}_{3} \mathrm{~N}_{4}-\mathrm{FeVO}_{4}-\mathrm{Fe} @ \mathrm{NH}_{2}$-biochar were reported by Kumar et al. ${ }^{35}$ Further, Zhang et al. synthesized $\mathrm{TiO}_{2} @ \mathrm{SiO}_{2}$ wrapped in a GO layer and state that the thin GO layer improves the charge separation. However, the thick layer of GO negatively affects the charge separation due to adsorbance or scattering of photon energy, and therefore the photocatalytic activity decreases. The similar masking effects of a thick carbon layer on photocatalysis also reported by $\mathrm{Wu}$ et $a .^{33}$

\subsection{Reducing band gap energy}

The band gap energy of semiconductor photocatalysts can be reduced by combining them with biochar. There are three major ways that have been reported in the literature for reducing the $\mathrm{TiO}_{2}-\mathrm{BSP}$ band gap: (1) sensitize the photocatalytic nanoparticle with another compound, (2) create a mid-gap energy state via doping non-metals such as N, S, C, O etc., and (3) formation of a local trapping state below the $\mathrm{TiO}_{2}$ conduction band. Usually, composites with small band gaps are used to sensitize the larger band gap semiconductor photocatalyst (Fig. 4a). Carbon can act as a sensitizer when doping with $\mathrm{TiO}_{2}$. The larger band gap of $\mathrm{TiO}_{2}$ can be sensitized by carbon doping, ${ }^{71}$ and therefore, the synthesized composite light sensitivity shifts from the UV region to the visible light region. For efficient sensitization, the conduction band of the sensitizer material should be placed higher than the conduction band of $\mathrm{TiO}_{2} \cdot{ }^{72}$ Another important aspect of the $\mathrm{TiO}_{2}-\mathrm{BSP}$ is the energy

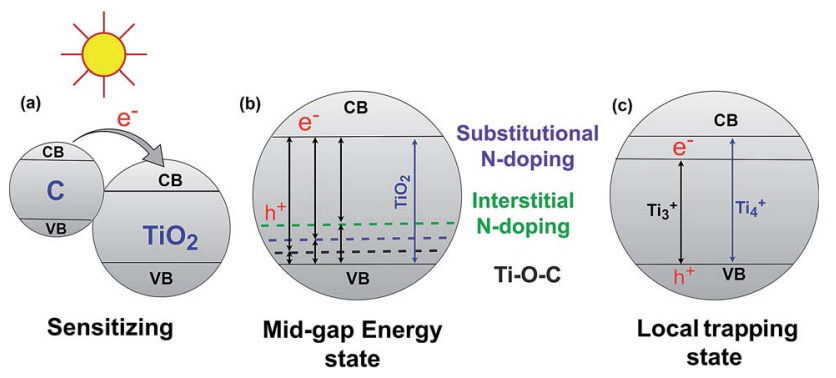

Fig. 4 Reduction of the $\mathrm{TiO}_{2}-\mathrm{BSP}$ band gap energy via sensitizing (a), forming a mid-gap energy state (b) and forming a local trapping state (c).

level of any impurities which form Ti-O-C bonds. ${ }^{73,74}$ This can create a delocalized state in the band gap of $\mathrm{TiO}_{2}$ without promoting charge carrier recombination. ${ }^{7}$ Furthermore, the incorporation of non-metals $\mathrm{N}$ or $\mathrm{S}$ with the $\mathrm{TiO}_{2}$-biochar significantly reduces the band gap of the composite by forming a mid-gap energy state. The excellent $\mathrm{N}$ - and S-doped biocharbased $\mathrm{TiO}_{2}$ semiconductor photocatalyst has been synthesized by Matos et al. ${ }^{29}$ and Bandosz et al. ${ }^{75}$ Due to $\mathrm{N}$ - and S-doping, the photocatalytic activity of the $\mathrm{TiO}_{2}$-composite increases up to 2 and 5 times more than the bare $\mathrm{TiO}_{2}$ photocatalyst. However, the photocatalytic activity of the composites is largely influenced by the texture and the content of $\mathrm{S}$ and $\mathrm{N}$ in the BSP. ${ }^{29,75}$ $\mathrm{N}$ - or S-doped BSPs not only reduce the energy gap of $\mathrm{TiO}_{2}$ but also act as electron shuttles from the $\pi$-orbital of the $\mathrm{N} / \mathrm{S}$-doped biochar to the conduction band of $\mathrm{TiO}_{2}$. The general procedures for reducing the band gap energy level by C-, substitutional $\mathrm{N}$-, and interstitial $\mathrm{N}$-doping in $\mathrm{TiO}_{2}$-biochar are illustrated in Fig. 4b. An excellent review article by Ansari et al. ${ }^{76}$ describes in detail the reduction of the band gap by $\mathrm{N}$-doping semiconductor photocatalysts. The local trapping state below $\mathrm{TiO}_{2}$ can also be produced in $\mathrm{TiO}_{2}$-biochar as shown in Fig. 4c. Lisowski et al. ${ }^{28}$ reported that a $\mathrm{TiO}_{2}$-biochar synthesized by an ultrasound method contained a local trapping state corresponding to $\mathrm{Ti}^{3+}$, which reduced the band gap of the BSP as well as enhancing the photocatalytic redox reaction.

\section{Applications of BSPs}

Many excellent new technologies can be understood via applications of BSPs, extending the existing wide range of photocatalytic nanoparticle applications. The most prominent application of BSPs is in the degradation of various hazardous organic pollutants in air and waterways. Furthermore, these BSPs can also be applied as film electrodes in solar cells, and as photocatalytic $\mathrm{H}_{2}$ generators in water splitting. The list of BSPs which have been synthesized and their applications in various fields are summarized in Table 1. Organic pollutants whose photodegradation by BSPs have been published at least once include phenol, ${ }^{28,77}$ bisphenol $\mathrm{A}^{38}$ methylene blue, ${ }^{29,33,37,75}$ safranin $\mathrm{T}^{41}$ anthraquinone, ${ }^{32}$ malic acid, ${ }^{59}$ sulfamethoxazole, ${ }^{25,30,34}$ methyl paraben, ${ }^{35}$ 2-chlorophenol, ${ }^{35}$ Reactive Yellow $39,{ }^{36}$ methyl orange ${ }^{39}$ in the liquid phase, and methanol in the 
gas phase.$^{28}$ Among the listed pollutants, methylene blue is the most commonly studied pollutant followed by sulfamethoxazole and phenol. The photodegradation of methylene blue by various BSPs ranges from $38 \%$ of $4.8 \mathrm{ppm}$ to $100 \%$ of $400 \mathrm{ppm}$, and the degradation time varies from $1.5 \mathrm{~h}$ to $4 \mathrm{~h}$. Phenol degradation capacity ranges from $64 \%$ to $75 \%$ of $50 \mathrm{ppm}$ within $4 \mathrm{~h}$, and sulfamethoxazole degradation capacity is $\sim 90 \%$ of $10 \mathrm{ppm}$ within $3 \mathrm{~h}$. The intermediate product analysis during photodegradation can give a clear understanding of the degradation pathway and evidence of mineralization. For example, the quantification of malic acid and methanol mineralization in water and air media by $\mathrm{TiO}_{2}-$ guanidine-(Ni,Co) $-\mathrm{Fe}_{2} \mathrm{O}_{4}$ (ref. 59) and $\mathrm{TiO}_{2}-\mathrm{SWP} 700,{ }^{28}$ respectively give a clear understanding of the pollutant photodegradation process. It shows that the overall conversion rate of malic acid and methanol was $60 \%$ and $88 \%$, respectively. Similarly, methyl paraben, 2-chlorophenol, and sulfamethoxazole photodegradation pathways were investigated by Kumar et al. $^{35}$ and Zhang et al., ${ }^{34}$ respectively. Biochar-supported photocatalysts are also applicable for the degradation of pollutants in the gaseous phase. Lisowski et al. ${ }^{28}$ passed a mixture of methanol and air $(0.9 \%+99.1 \%$ vol $)$ at a flow rate of $25 \mathrm{~cm}^{3} \mathrm{~min}^{-1}$ through an author-designed BSPcontaining photo-reactor. The reaction output products were measured online by gas chromatography and offline by GC-MS under conditions both with and without UV light illumination. The UV illumination and presence of $\mathrm{TiO}_{2}-\mathrm{SWP700}$ significantly mineralized methanol into $\mathrm{CO}_{2}$ and methyl formate. Besides, organic pollutant degradation, BSPs also show good performance as film electrodes in solar cells and as $\mathrm{H}_{2}$ producers in water splitting. Matos et al. ${ }^{31}$ synthesized $\mathrm{TiO}_{2}-\mathrm{C}$ hollow spheres via a solvothermal and post-calcination process of $\mathrm{TiO}_{2}-$ chitosan/furfural/saccharose precursors. The obtained BSPs were successfully applied as film electrodes in a solar cell under visible light catalysis. Furthermore, Matos et al. synthesized ${ }^{78} \mathrm{Au}-\mathrm{TiO}_{2}$ biochar through multi-step heating and slurry methods and applied them for $\mathrm{H}_{2}$ production from water. The author states that the biochar-supported $\mathrm{Au}-\mathrm{TiO}_{2}$ performs 3 times better photocatalysis than the commercial semiconductor photocatalyst and the $\mathrm{O}$ functional groups of biochar enhance the photocatalytic $\mathrm{H}_{2}$ production. It also worth mentioning that BSPs possess high recyclability during organic pollutant photodegradation. In most cases, their organic removal capacity decreased only $2-10 \%$ after recycling the BSPs 3-6 times (Table 1 ), which indicates the higher stability, recyclability and cost effectiveness of the composites.

\section{BSP-driven photodegradation mechanisms}

\subsection{Adsorption}

Incorporating catalytic nanoparticles with biochar can increase the BSP affinity for adsorbing a variety of organic pollutants in an aqueous medium. A higher adsorption potentiality of BSPs enhances photodegradation of organic pollutants to a greater extent than bare nanoparticles. ${ }^{33}$ Colmenares et al. ${ }^{77}$ reported that similar Ti-doping with different biochar components significantly varies their pollutant removal competency. As shown in Fig. 5, 15Ti/GO, 15 Ti/Norit, and 15Ti/Starbon exhibit different phenol reduction competencies, even though a similar synthetic process and amount of Ti has been applied in their syntheses. The higher phenol reduction competency shown by $15 \mathrm{Ti} /$ Starbon is due to its higher adsorption competency, its hybrid interphase proximity, and the presence of a highlycrystalline anatase phase.

A similar example of higher photodegradation due to higher adsorption capacity of the composite was reported by Luo et al. ${ }^{38}$ The commercial P25 and author-synthesized $\mathrm{TiO}_{2}-$ WC-450 exhibits a similar ratio of anatase and rutile $\mathrm{TiO}_{2}$ (80:20). However, $\mathrm{TiO}_{2}-\mathrm{WC}-450$ exhibits 1.85 times higher removal of bisphenol A than $\mathrm{P} 25$, which was due to higher adsorption and mass transfer competency of biocharsupported $\mathrm{TiO}_{2}$. The biochar contents in the photocatalyst play a key role in the adsorption as well as in photodegradation. Due to surface adsorption capabilities, organic pollutants can deposit on the catalyst surface before transfer to the decomposition center. Therefore, the reactive oxygen species generated due to light illumination do not need to migrate so far from the activation site. However, some fractions of organic pollutants are adsorbed on the catalyst without coming into contact with $\mathrm{TiO}_{2}$. Those particles also undergo attack by ROS produced on the surface of $\mathrm{TiO}_{2}$. It is assumed that the ROS could diffuse over a sub-millimeter distance from the activation site to the reactant site located on the surface of the composites. ${ }^{28}$ The synergistic effect of adsorption and photodegradation give rise to an efficient photodegradation process. ${ }^{34,60}$ Kumar et al. ${ }^{35}$ reported that the simultaneous adsorption-photodegradation allow high and rapid degradation of methyl paraben (MeP) and 2-chlorophenol (2-CP) due to the immediate attack of ROS after being adsorbed onto the photocatalyst. However, adsorption followed by photodegradation reduces the degradation competency due to covering most of the surface active sites with the pollutants. ${ }^{35}$

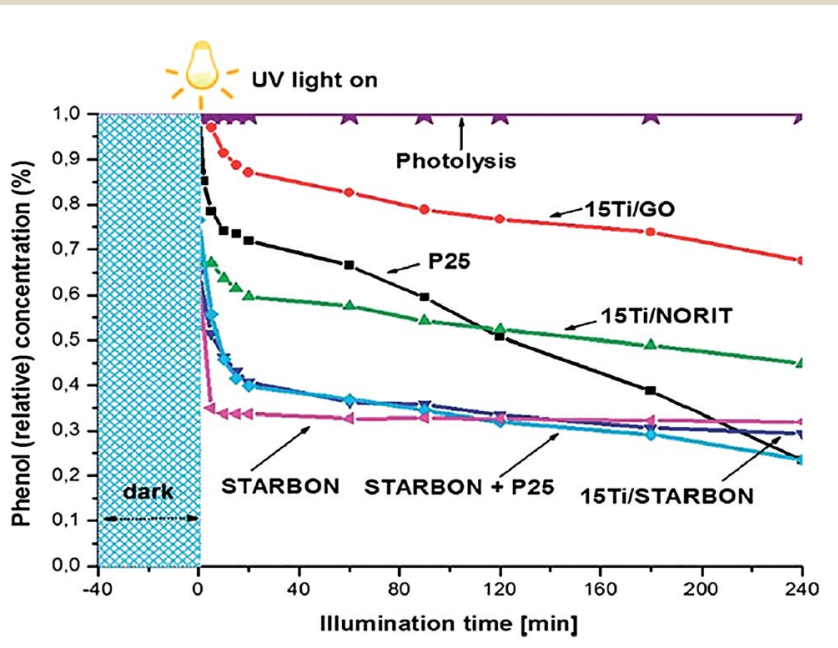

Fig. 5 Photocatalytic degradation of phenol by different photocatalysts. Reproduced with permission from ref. 77 . 


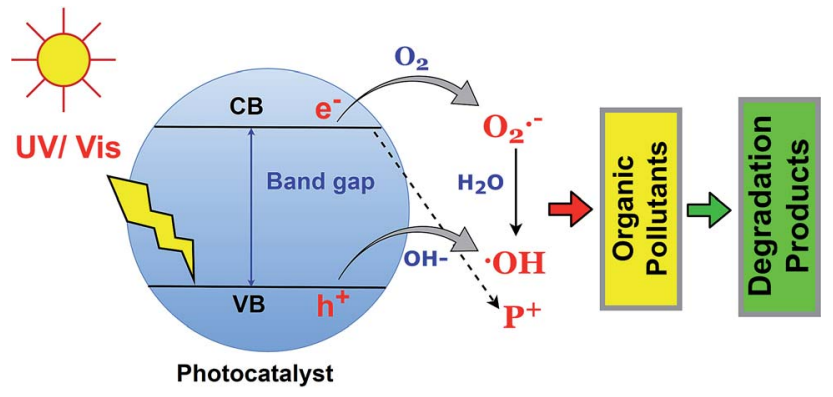

Fig. 6 Fundamentals of organic pollutant photodegradation by ROS.

\subsection{Photodegradation}

The photodegradation of organic pollutants using BSPs is the function of reactive oxygen species such as ${ }^{\circ} \mathrm{OH}$ and $\mathrm{O}_{2}{ }^{-}$, and $\mathrm{h}^{+}$. Identification of specific ROS functions during photocatalysis is essential to understand the photocatalytic redox reaction. ${ }^{79}$ Generally, the photocatalyst adsorbed a photon with an energy which is equal to or less than the band gap, and excited the electron in the valence band to the conduction band. Therefore, an electron-hole is created in the upper edge of the valence band and an electron occupies a phase on the lower edge of the conduction band. Later on, the $\mathrm{h}^{+}$and $\mathrm{e}^{-}$can either recombine or diffuse to the acceptor. The $\mathrm{e}_{(\mathrm{CB})}{ }^{-}$and $\mathrm{h}_{(\mathrm{VB})}{ }^{+}$can scavenge the $-\mathrm{OH}, \mathrm{O}_{2}$, and $\mathrm{H}_{2} \mathrm{O}$ molecules in the liquid phase and successively produce ${ }^{\circ} \mathrm{OH}$ and $\mathrm{O}_{2}{ }^{-}$- species, which further react with organic pollutants leading to complete mineralization. The fundamentals of organic pollutant photodegradation by ROS are illustrated in Fig. 6. The role of specific ROS in various pollutant photodegradation using various BSPs is different. Recently, many researchers have been investigating the mechanisms of organic pollutant photodegradation using specific ROS identification and degradation product analysis. In most cases, ROS scavenging by different inhibitors and mass spectroscopic methods have been used..$^{33,80,81}$ A comprehensive list of different ROS-function identification processes for organic pollutant photodegradation by BSPs is summarized in Table 2. Scavenger benzoquinone and TEMPOL for $\mathrm{O}_{2}{ }^{\cdot-}$, ammonium oxalate, triethanolamine, and EDTA $2 \mathrm{Na}$ for $\mathrm{h}^{+}$, and tert-butyl alcohol and isopropanol for ${ }^{\circ} \mathrm{OH}$ identification are very popular in ROS scavenging experiments. As shown in Table 2 , the organic pollutants were degraded by the action of multiple ROS. The ranking was made based on specific ROS pollutant removal competencies determined by scavenging experiments. An excellent review article has recently been published with extensive discussion about the generation and detection process of ROS in photocatalysts. ${ }^{79}$ According to Nosaka and Nosaka, MCLA chemiluminescence, lucigenin chemiluminescence, $1270 \mathrm{~nm}$ emission and coumarin fluorescence probing are the most recommended methods for the detection of $\mathrm{O}_{2}{ }^{--}, \mathrm{H}_{2} \mathrm{O}_{2}, \mathrm{O}_{2}$, and ${ }^{\circ} \mathrm{OH}$.

\subsection{Ozonization}

Ozonization during photocatalytic degradation of organic pollutants by BSPs influences reaction rate and speed. The combined mechanisms of adsorption, photodegradation, and ozonization enhance organic pollutant removal competencies to a greater extent. ${ }^{35}$ During the combined treatment, ozone leads to the production of ${ }^{\circ} \mathrm{OH}$ and $\mathrm{H}_{2} \mathrm{O}_{2}$ due to exposure to photon energy. Meanwhile, the electron which is unable to recombine due to the heterojunction in the BSP, substantially increases the production of $\mathrm{O}_{2}{ }^{-}-$and ${ }^{\circ} \mathrm{OH}$ by reaction with $\mathrm{O}_{2}$ and $\mathrm{H}_{2} \mathrm{O}_{2}$. Therefore, the reaction rate between ROS and pollutants increases, which results in a rapid and high reduction of pollutants. Furthermore, Fe-doped BSPs can give Fentonlike reaction competency. Our synthesized biochar-supported photocatalyst $\left(\mathrm{N}-\mathrm{TiO}_{2}-\mathrm{Fe}_{3} \mathrm{O}_{4}\right.$-biochar $)$ exhibited very high methylene blue reduction under visible light irradiation due to injecting $\mathrm{H}_{2} \mathrm{O}_{2}$ in the reaction system. Similarly, graphene oxide-encapsulated $\mathrm{Fe}_{3} \mathrm{O}_{4}-\mathrm{TiO}_{2}$ spheres synthesized by Yang et al., ${ }^{8}$ shows good Fenton-like degradation competency of methylene blue under visible light irradiation as a result of rapid redox reactions between $\mathrm{Fe}^{2+}$ and $\mathrm{Fe}^{3+}$.

Table 2 Responsible ROS and their function identification processes in different organic pollutant photocatalysis by BSPS

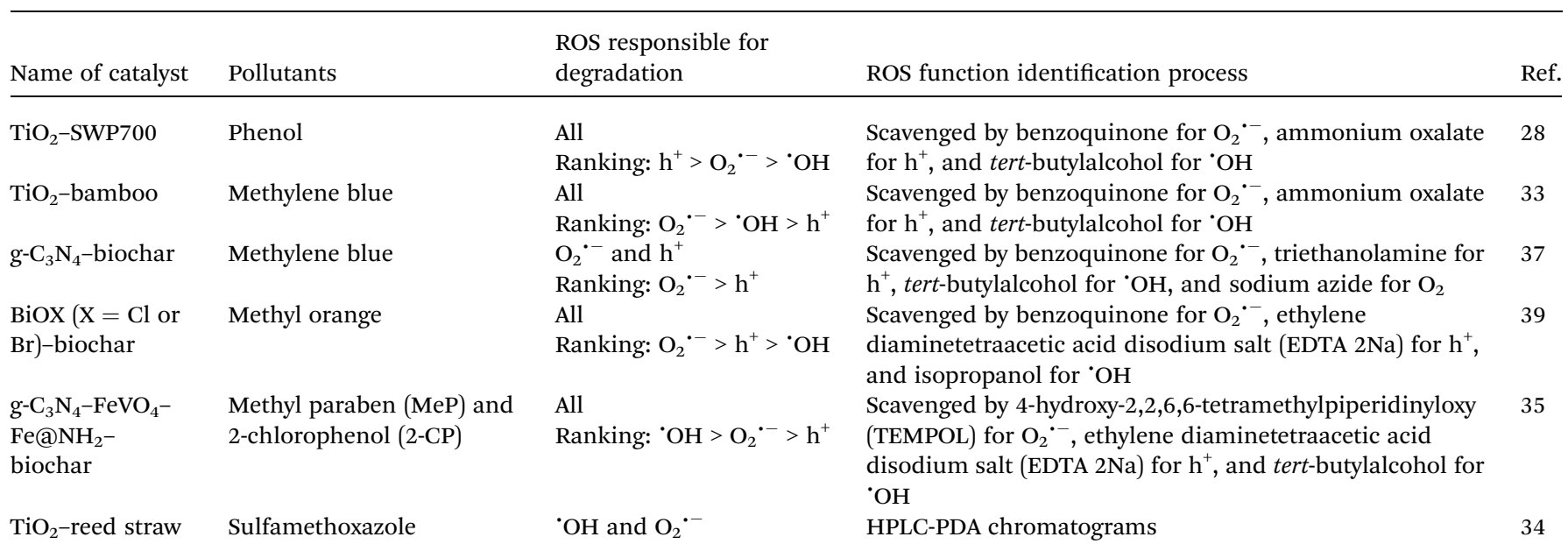


Finally, it is also required to mention that the dose of organic pollutants and catalyst has a significant influence on the photodegradation process. Unlike adsorption of pollutants, photodegradation and simultaneous adsorption-photodegradation do not proportionally increase with the concentration of catalyst dose. Therefore, a specific dose of catalyst or pollutants may require determining to achieve optimum pollutant degradation. High concentrations of pollutants can prevent the light being able to reach the reactant photocatalyst. Therefore, the production of ROS can be reduced. Similarly, the dose of a catalyst has an optimum limit to reach maximum photocatalysis. An excessive photocatalyst dose can cause light scattering, which hinders the specific activity of the photocatalyst. ${ }^{\mathbf{8 2} 83}$ Moreover, the number of active sites on the reactants can be reduced due to agglomeration of homogeneous particles at a high dosage. ${ }^{\mathbf{8 4}}$ Therefore, an optimum dose of BSPs and pollutants should be measured to achieve optimum performance of the photocatalyst.

\section{Conclusion and outlook}

This review gives a detailed overview of the recent progress in the field of BSPs, summarized their synthetic processes and physicochemical characteristics, explicitly discussed the biochar role in the BSP, and pollutant photodegradation. It has revealed that the incorporation of transition metals/catalytic nanoparticles and/or non-metals with biochar templates are effective techniques to produce high-performance semiconductor photocatalysts. The catalytic nanoparticles can easily be incorporated with biochar through sol-gel, solvothermal, ultrasound, thermal polycondensation, and hydrolysis methods. The photocatalytic performance of BSPs significantly increases due to the unique physicochemical properties of biochar. Biochar can support a large number of nanoparticles, increase the number of active sites on the photocatalyst, act as an electron reservoir which conducts away the electron from the $\mathrm{e}^{-} / \mathrm{h}^{+}$, shuttle electrons through a graphene-like skeleton, improve charge separation, and reduce band gap energy by carbon or other non-metal doping with catalytic nanoparticles. Unlike bare catalytic nanoparticles, BSP adsorption capacity during photocatalysis enhances photodegradation performance. Simultaneous adsorption and photodegradation perform rapid and higher organic pollutant degradation due to the immediate attack of nearby ROS. Moreover, BSPs are more convenient to recover from aqueous solution than bare CNPs. Up until now, BSPs have mainly been applied for the photodegradation of organic pollutants in the aqueous phase. However, only a small portion of research focuses on BSP applications for clean energy production, air pollutant treatment, and anti-bacterial sterilization. It has already been proven that BSPs are an effective material for photocatalytic applications due to their cost-effectiveness, sustainability, and higher visible light photoactivity. However, there are still a number of aspects that need to be considered for the future development of BSPs.

- Prior to the synthesis of BSPs, it is worth considering the cost-effectiveness and the availability of natural biomass, a facile synthetic process to incorporate catalytic nanoparticles, and an efficient modification technique to enhance optical properties in the visible light region.

- Applying a wide variety of biomass and nanoparticles in the synthesis of BSPs and examining the synergistic effects of biochar and catalytic nanoparticle incorporation.

- The key controlling factors that stimulate BSP performance characteristics should be investigated such as heating operating conditions, selection and ratio of biomass and nanoparticles, and incorporation processes.

- Various hazardous waste materials such as sewage sludge, manure, and various organic and inorganic municipal waste materials can be used in the synthesis of BSPs. The waste material contains various catalytic nanoparticles that could improve the BSP photoresponse. ${ }^{85}$ Furthermore, natural polysaccharides have unique physicochemical properties such as formation of gels, flocking, and complete dissolution in water, which are beneficial to the incorporation of various compounds. This is a novel, sustainable and environmentally friendly way to manage municipal waste.

- Introduction of BSPs in many other photocatalytic applications and investigation of the photocatalysis mechanisms and performance.

\section{Conflicts of interest}

There are no conflicts to declare.

\section{Acknowledgements}

This work was supported by the National Basic Research Program of China (973 Program, 2014CB238903) and the National Natural Science Foundation of China (No. 41672144, 41173032, and 41373110). We thank the editors and anonymous reviewers for giving us many constructive comments that significantly improved the paper.

\section{References}

1 WWAP (United Nations World Water Assessment Programme), The United Nations World Water Development Report 2017: Wastewater, The Untapped Resource, UNESCO, Paris, 2017.

2 J. C. Colmenares, R. S. Varma and P. Lisowski, Green Chem., 2016, 18, 5736-5750.

3 G. Jayanthi Kalaivani and S. K. Suja, Carbohydr. Polym., 2016, 143, 51-60.

4 J. Schneider, M. Matsuoka, M. Takeuchi, J. Zhang, Y. Horiuchi, M. Anpo and D. W. Bahnemann, Chem. Rev., 2014, 114, 9919-9986.

5 Y. Yamada, S. Shikano and S. Fukuzumi, J. Phys. Chem. C, 2013, 117, 13143-13152.

6 M. T. Uddin, O. Babot, L. Thomas, C. Olivier, M. Redaelli, M. D’Arienzo, F. Morazzoni, W. Jaegermann, N. Rockstroh, H. Junge and T. Toupance, J. Phys. Chem. C, 2015, 119, 7006-7015.

7 J. Chung, J. W. Chung and S.-Y. Kwak, Phys. Chem. Chem. Phys., 2015, 17, 17279-17287. 
8 X. Yang, W. Chen, J. Huang, Y. Zhou, Y. Zhu and C. Li, Sci. Rep., 2015, 5, 10632.

9 P. Kumar, C. Joshi, N. Labhsetwar, R. Boukherroub and S. L. Jain, Nanoscale, 2015, 7, 15258-15267.

10 A. Gołąbiewska, W. Lisowski, M. Jarek, G. Nowaczyk, A. Zielińska-Jurek and A. Zaleska, Appl. Surf. Sci., 2014, 317, 1131-1142.

11 M. Cui, S. Pan, Z. Tang, X. Chen, X. Qiao and Q. Xu, Chem. Speciation Bioavailability, 2017, 29, 60-69.

12 P. Wang, P.-S. Yap and T.-T. Lim, Appl. Catal., A, 2011, 399, 252-261.

13 K. Kalantari, M. Kalbasi, M. Sohrabi and S. J. Royaee, Ceram. Int., 2016, 42, 14834-14842.

14 H. Tian, J. Ma, K. Li and J. Li, Ceram. Int., 2009, 35, 12891292.

15 I. Delidovich, K. Leonhard and R. Palkovits, Energy Environ. Sci., 2014, 7, 2803-2830.

16 S.-H. Kong, S.-K. Loh, R. T. Bachmann, S. A. Rahim and J. Salimon, Renewable Sustainable Energy Rev., 2014, 39, 729-739.

17 J. Han, X. Wang, J. Yue, S. Gao and G. Xu, Fuel Process. Technol., 2014, 122, 98-106.

18 E. Gregorová, W. Pabst and I. Bohačenko, J. Eur. Ceram. Soc., 2006, 26, 1301-1309.

19 C. D. Tran, S. Duri, A. Delneri and M. Franko, J. Hazard. Mater., 2013, 252-253, 355-366.

20 D. H. K. Reddy and S.-M. Lee, Adv. Colloid Interface Sci., 2013, 201-202, 68-93.

21 S. Mallick, S. Sharma, M. Banerjee, S. S. Ghosh, A. Chattopadhyay and A. Paul, ACS Appl. Mater. Interfaces, 2012, 4, 1313-1323.

22 W.-J. Liu, H. Jiang and H.-Q. Yu, Chem. Rev., 2015, 115, 12251-12285.

23 Y. Xia and R. C. Larock, Green Chem., 2010, 12, 1893-1909.

24 S. Dutta, A. Bhaumik and K. C.-W. Wu, Energy Environ. Sci., 2014, 7, 3574-3592.

25 J. R. Kim and E. Kan, J. Environ. Manage., 2016, 180, 94-101.

26 M. Dahl, Y. Liu and Y. Yin, Chem. Rev., 2014, 114, 98539889.

27 H.-P. Jing, C.-C. Wang, Y.-W. Zhang, P. Wang and R. Li, RSC Adv., 2014, 4, 54454-54462.

28 P. Lisowski, J. C. Colmenares, O. Mašek, W. Lisowski, D. Lisovytskiy, A. Kamińska and D. Łomot, ACS Sustainable Chem. Eng., 2017, 5, 6274-6287.

29 J. Matos, M. Hofman and R. Pietrzak, Carbon, 2013, 54, 460471.

30 Q. Liu, F. Yang, X. Sun, Z. Liu and G. Li, J. Mater. Cycles Waste Manage., 2017, 19, 134-143.

31 J. Matos, P. Atienzar, H. García and J. C. Hernández-Garrido, Carbon, 2013, 53, 169-181.

32 A. Khataee, B. Kayan, P. Gholami, D. Kalderis and S. Akay, Ultrason. Sonochem., 2017, 39, 120-128.

33 F. Wu, W. Liu, J. Qiu, J. Li, W. Zhou, Y. Fang, S. Zhang and X. Li, Appl. Surf. Sci., 2015, 358, 425-435.

34 H. Zhang, Z. Wang, R. Li, J. Guo, Y. Li, J. Zhu and X. Xie, Chemosphere, 2017, 185, 351-360.
35 A. Kumar, A. Kumar, G. Sharma, M. Naushad, F. J. Stadler, A. A. Ghfar, P. Dhiman and R. V. Saini, J. Cleaner Prod., 2017, 165, 431-451.

36 A. Khataee, B. Kayan, P. Gholami, D. Kalderis, S. Akay and

L. Dinpazhoh, Ultrason. Sonochem., 2017, 39, 540-549.

37 L. Pi, R. Jiang, W. Zhou, H. Zhu, W. Xiao, D. Wang and X. Mao, Appl. Surf. Sci., 2015, 358, 231-239.

38 L. Luo, Y. Yang, M. Xiao, L. Bian, B. Yuan, Y. Liu, F. Jiang and X. Pan, Chem. Eng. J., 2015, 262, 1275-1283.

39 M. Li, H. Huang, S. Yu, N. Tian, F. Dong, X. Du and Y. Zhang, Appl. Surf. Sci., 2016, 386, 285-295.

40 Y. Li, S. Zhang, Q. Yu and W. Yin, Appl. Surf. Sci., 2007, 253, 9254-9258.

41 X. Cai, J. Li, Y. Liu, Z. Yan, X. Tan, S. Liu, G. Zeng, Y. Gu, X. Hu and L. Jiang, J. Chem. Technol. Biotechnol., 2018, 93, 783-791.

42 Y. Wang, M. Dong, M. Guo, X. Wang, J. Zhou, J. Lei, C. Guo and C. Qin, Mater. Sci. Eng., C, 2017, 77, 293-299.

43 V. Normand, D. L. Lootens, E. Amici, K. P. Plucknett and P. Aymard, Biomacromolecules, 2000, 1, 730-738.

44 Z. Zhang, Z. Luo, Z. Yang, S. Zhang, Y. Zhang, Y. Zhou, X. Wang and X. Fu, RSC Adv., 2013, 3, 7215-7218.

45 M. Pelaez, B. Baruwati, R. S. Varma, R. Luque and D. D. Dionysiou, Chem. Commun., 2013, 49, 10118-10120.

46 J. Song, X. Wang, J. Huang, J. Ma, X. Wang, H. Wang, R. Ma, P. Xia and J. Zhao, Electrochim. Acta, 2016, 222, 1-11.

47 J. Matos, A. García, L. Zhao and M. M. Titirici, Appl. Catal., A, 2010, 390, 175-182.

48 J. Matos and A. Corma, Appl. Catal., A, 2011, 404, 103-112.

49 J. Matos, A. Borodzinski, A. M. Zychora, P. Kedzierzawski,

B. Mierzwa, K. Juchniewicz, M. Mazurkiewicz and

J. C. Hernández-Garrido, Appl. Catal., B, 2015, 163, 167-178.

50 A. E. Creamer, B. Gao and M. Zhang, Chem. Eng. J., 2014, 249, 174-179.

51 D. Woolf, J. E. Amonette, F. A. Street-Perrott, J. Lehmann and S. Joseph, Nat. Commun., 2010, 1, 56.

52 X. Tan, Y. Liu, Y. Gu, Y. Xu, G. Zeng, X. Hu, S. Liu, X. Wang, S. Liu and J. Li, Bioresour. Technol., 2016, 212, 318-333.

53 J. Yang, Y. Zhao, S. Ma, B. Zhu, J. Zhang and C. Zheng, Environ. Sci. Technol., 2016, 50, 12040-12047.

54 J. Yang, S. Han, H. Zheng, H. Dong and J. Liu, Carbohydr. Polym., 2015, 123, 53-66.

55 H. Jonassen, A.-L. Kjøniksen and M. Hiorth, Biomacromolecules, 2012, 13, 3747-3756.

56 M. Rinaudo, Prog. Polym. Sci., 2006, 31, 603-632.

57 M. Vakili, M. Rafatullah, B. Salamatinia, A. Z. Abdullah, M. H. Ibrahim, K. B. Tan, Z. Gholami and P. Amouzgar, Carbohydr. Polym., 2014, 113, 115-130.

58 M. Zhang, Y. Liu, T. Li, W. Xu, B. Zheng, X. Tan, H. Wang, Y. Guo, F. Guo and S. Wang, RSC Adv., 2015, 5, 46955-46964.

59 A. M. Balu, B. Baruwati, E. Serrano, J. Cot, J. Garcia-Martinez, R. S. Varma and R. Luque, Green Chem., 2011, 13, 2750-2758. 60 X. Wang, Y. Liu, Z. Hu, Y. Chen, W. Liu and G. Zhao, J. Hazard. Mater., 2009, 169, 1061-1067.

61 M. A. Nawi, A. H. Jawad, S. Sabar and W. S. W. Ngah, Desalination, 2011, 280, 288-296. 
62 L. Yu, Y. Yuan, J. Tang, Y. Wang and S. Zhou, Sci. Rep., 2015, $5,16221$.

63 W. Xu, J. J. Pignatello and W. A. Mitch, Environ. Sci. Technol., 2013, 47, 7129-7136.

64 X. Yu, W. Gong, X. Liu, L. Shi, X. Han and H. Bao, J. Hazard. Mater., 2011, 198, 340-346.

65 X.-L. Wu, T. Wen, H.-L. Guo, S. Yang, X. Wang and A.-W. Xu, ACS Nano, 2013, 7, 3589-3597.

66 Y. Yao and F. Wu, ACS Appl. Mater. Interfaces, 2017, 9, 3190731912.

67 L. Klüpfel, M. Keiluweit, M. Kleber and M. Sander, Environ. Sci. Technol., 2014, 48, 5601-5611.

68 J. M. Saquing, Y.-H. Yu and P. C. Chiu, Environ. Sci. Technol. Lett., 2016, 3, 62-66.

69 Y.-J. Xu, Y. Zhuang and X. Fu, J. Phys. Chem. C, 2010, 114, 2669-2676.

70 J. Shi, Chem. Rev., 2013, 113, 2139-2181.

71 W. Wang, P. Serp, P. Kalck and J. L. Faria, J. Mol. Catal. A: Chem., 2005, 235, 194-199.

72 Y. Qu and X. Duan, Chem. Soc. Rev., 2013, 42, 2568-2580.

73 Y. H. Ng, S. Ikeda, M. Matsumura and R. Amal, Energy Environ. Sci., 2012, 5, 9307-9318.

74 Y. Li, D.-S. Hwang, N. H. Lee and S.-J. Kim, Chem. Phys. Lett., 2005, 404, 25-29.
75 T. J. Bandosz, J. Matos, M. Seredych, M. S. Z. Islam and R. Alfano, Appl. Catal., A, 2012, 445-446, 159-165.

76 S. A. Ansari, M. M. Khan, M. O. Ansari and M. H. Cho, New J. Chem., 2016, 40, 3000-3009.

77 J. C. Colmenares, P. Lisowski and D. Lomot, RSC Adv., 2013, 3, 20186-20192.

78 J. Matos, Top. Catal., 2016, 59, 394-402.

79 Y. Nosaka and A. Y. Nosaka, Chem. Rev., 2017, 117, 1130211336.

80 A. Molla, M. Sahu and S. Hussain, J. Mater. Chem. A, 2015, 3, 15616-15625.

81 Q. Lu, Y. Zhang and S. Liu, J. Mater. Chem. A, 2015, 3, 85528558.

82 W. Liu, Q. Hu, F. Mo, J. Hu, Y. Feng, H. Tang, H. Ye and S. Miao, J. Mol. Catal. A: Chem., 2014, 395, 322-328.

83 X. Wang, P. Wu, Y. Lu, Z. Huang, N. Zhu, C. Lin and Z. Dang, Sep. Purif. Technol., 2014, 132, 195-205.

84 M. Ahmadi, H. Ramezani Motlagh, N. Jaafarzadeh, A. Mostoufi, R. Saeedi, G. Barzegar and S. Jorfi, J. Environ. Manage., 2017, 186, 55-63.

85 H. Huang, T. Yang, F. Lai and G. Wu, J. Anal. Appl. Pyrolysis, 2017, 125, 61-68. 